\title{
Supporting Information for \\ Covalent Functionalization for Biomolecular Recognition on Vertically Aligned Carbon Nanofibers
}

Sarah E. Baker, Kiu-Yuen Tse, Eve Hindin, Beth M. Nichols, Tami Lasseter Clare, and Robert J. Hamers

\author{
Department of Chemistry \\ University of Wisconsin-Madison \\ 1101 University Avenue \\ Madison, WI 53706
}

This supporting information depicts X-ray photoelectrons spectroscopy data confirming the photochemical functionalization of carbon nanofibers with the tBOCproteced 10-aminodec-1-ene, as depicted in panel (a) below. Panels (b) and (c) show $\mathrm{N}(1 \mathrm{~s})$ and $\mathrm{O}(1 \mathrm{~s})$ XPS data on bare fibers (i.e., fibers immediately after growth) and on fibers after photochemical modification with the tBOC-proteced 10-aminodec-1-ene. These spectra were normalized such that the area of the $C(1 s)$ peaks (not shown) are the same for both samples.

The N(1s) spectrum (b) shows that the bare, as-grown nanofibers have a significant N(1s) intensity, yielding two peaks at 397.9 and $400.1 \mathrm{eV}$. These peaks arise from incorporation of nitrogen from the ammonia during growth. After functionalization, the $\mathrm{N}(1 \mathrm{~s})$ shows only a single peak centered at $\sim 400.2 \mathrm{eV}$. This peak is nearly identical in energy to that reported previously for this same molecule photochemically linked to the $\operatorname{Si}(001)$ surface. ${ }^{1}$

The $\mathrm{O}(1 \mathrm{~s})$ region shows some oxygen in the original nanofibers, as two peaks at 531.8 and $533.1 \mathrm{eV}$. After functionalization, these two peaks are reduced to approximately $60 \%$ of their original intensity, and a new shoulder appears at $530.0 \mathrm{eV}$. The attenuation of the peaks at 531.8 and $533.1 \mathrm{eV}$ is attributed to inelastic scattering of electrons that arise from oxygen in the bulk nanofibers. The $\sim 40 \%$ attenuation is consistent with the expected inelastic scattering of electrons that arise from $\mathrm{O}$ in the bulk nanofibers but after functionalization must propagate through the monolayer film of tBOC-proteced 10-aminodec-1-ene. The new shoulder at $530 \mathrm{eV}$ has a significantly lower binding energy; this is consistent with its assignment as the carbonyl oxygen of the tBOC protecting group, which is also similar to the environment found in poly(urethane) polymers. $^{2,3}$ In these materials, the transfer of electron density from carbon to oxygen increases the binding energy of the carbon atom and decreases the binding energy of the oxygen, leading to relatively low $\mathrm{O}(1 \mathrm{~s})$ binding energies.

1) Strother, T.; Hamers, R. J.; Smith, L. M., Nucl. Acids Res. 2000, 28, 3535-3541.

2) Tannenbaum, R.; Hakanson, C.; Zeno, A.; Tirrell, M., Langmuir 2002, 18, 5592-5599.

3) G. Beamson and D. Briggs, "High Resolution XPS of Organic Polymers - The Scienta ESCA300 Database", J. Wiley, 1992. 


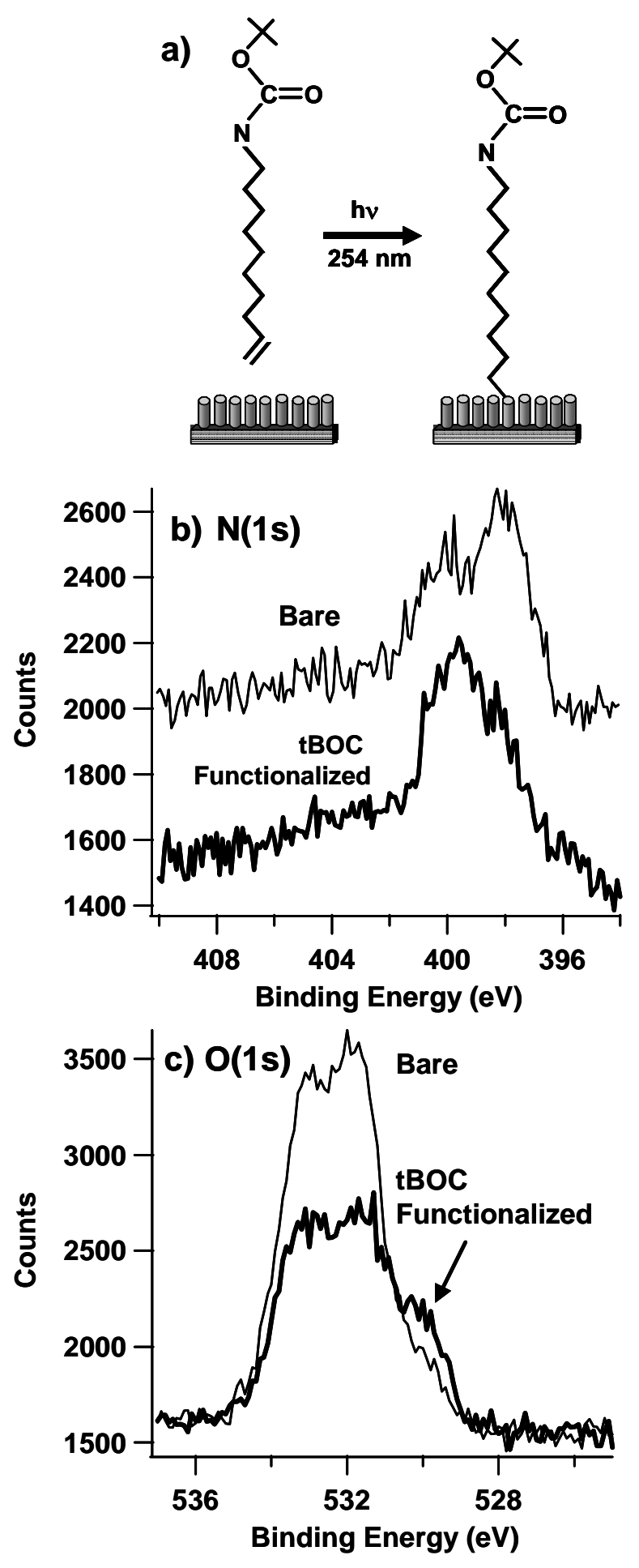

